\title{
Short Period Forecasting of PV Output using Hemispherical Photograph
}

\author{
Yusuke Imanishi ${ }^{1}$, Ryo Watanabe ${ }^{1}$, Yusuke Hida ${ }^{1}$, Ryuichi Yokoyama $^{1}$, Kenji Iba ${ }^{2}$ \\ ${ }^{1}$ Graduate School of Environment and Energy EngineeringWaseda University \\ Campus of Nishi - Waseda, 55 ${ }^{\text {th }}$ Building S-Wing 705 3-4-1 Okubo, Shinjuku-ku, Tokyo 169-8555(Japan) \\ Phone/Fax number: +81-3- 5286-3126/+81-3-3209-6323 \\ e-mail: jike-yoshino@fuji.waseda.jp, q6-yw@asagi.waseda.jp, yusuke.h@akane.waseda.jp, \\ yokoyama-ryuichi@waseda.jp \\ ${ }^{2}$ Department of Electrical Engineering Meisei University, \\ Campas of Hino, 2-1-1 Hodokubo, Hino-shi, Tokyo 191-8506 (Japan) \\ Phone/Fax number: +81-42-591-5426/+81-42-591-5426, iba@ee.meisei-u.ac.jp
}

\begin{abstract}
The spread and promotion of renewable energy is a worldwide subject. However, the intermittency of renewables, may cause troubles to power system operation. One of effective countermeasures to mitigate the intermittency is the battery energy storage system (BESS). The accurate forecasting of renewable energy output is also indispensable to reduce the capacity of BESS. In this paper, a new approach to forecast a photovoltaic power (PV) output using hemispherical photograph is presented. The photos are taken by fish-eye lens to get the whole sky cloud image data. The movement of cloud around the sun is analyzed and modeled to forecast the future output of PV. The numerical test results show a potential ability to forecast.
\end{abstract}

\section{Key words}

Photovoltaic power, short-forecasting, correlation coefficient, hemispherical photograph.

\section{Introduction}

In recent years, renewable energy such as wind power and solar power(PV) is regarded as one of the most promissing renewabels from the background, such as global warming and fossil fuel depletion. Especially, the total capacity of PV generation is expected to be $53 \mathrm{GW}$ by 2030 in Japan. However, the output of PV depends on weather. The nature of intermittency of renewables may cause both frequency and voltage fluctuations. It is serious concern whether we can conduct the stable operation of a power system or not when massive PVs are installed.

In order to overcome the difficulties some energy storage systems may be utilized. Among energy storage systems, battery energy system (BESS) seems to be the most promising. The cost of BESS, however, will push up the total cost of the PV system. The BESS is used to level the output of PV, therefore the accurate forecasting of PV's output can reduce the required capacity of BESS. However, the measure to predict the short-period fluctuation has not been established yet. This is because the current weather radar can not capture small cloud movement and change in a short time. Besides, the analysis of radar is not sufficient to predict the weather condition of the local PV site.

The most severe fluctuation of PV output will be caused by cloud when the weather is fine. It is a good idea to observe the movement of cloud for forecasting PV output. Although this idea is already proposed in some papers[1], it is hard to model and evaluate the movement of cloud.

In this paper a basic analysis to forecast PV output is tested and evaluated. Hemispherical photographs taken by fish-eye lens are used to get the whole sky cloud image data. The photo can be taken easily on site of a PV plant. The image data is decomposed into three elements, i.e., hue, saturation, brightness. The correlation between PV output and picture elements is calculated. If PV output shows strong relationship with some pixels of a photo that was taken 20 minute before, we can say that we can forecast the PV output based on the pixels data. In this paper, these correlations are calculated using field tests' data. The test results show that the correlations can be used to forecast the PV output.

\section{Data Analysis of Cloud Photo}

It is well-known that amount of global solar radiation affects PV output significantly. Since the amount of global solar radiation is blocked by clouds, it is important to predict the movement of clouds. The soft cloud, however, change the shape, thickness, speed and direction easily. Since it is very difficult to predict the movement of cloud directly, the correlations between the pixels of photo data and PV output is analyzed.

A. The image data analysis

The photo by fish-eye lens surely catches the sun in the circle frame. There is a difficulty to identify cloud and halation. Both "cloud" and "halation of the clear sky near the sun" give similar white images in the photo. An 


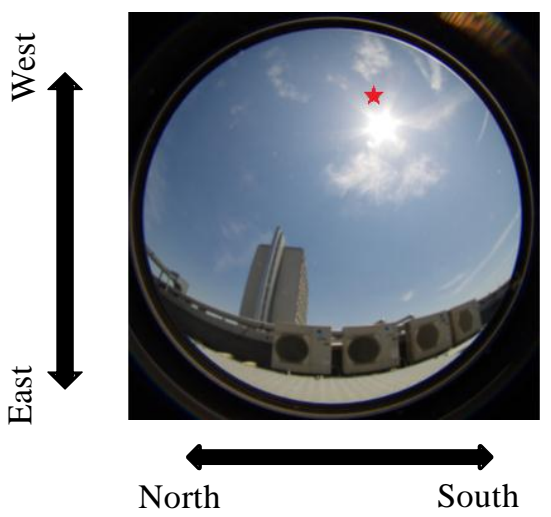

Fig.1 Cloud observation by hemispherical (fish-eye) photo

example of the hemispherical (fish-eye) photo is shown in Fig. 1

In order to distinguish the two conditions, the picture images are divided into three elements, such as saturation, hue and brightness. Three elements of the images in a point near the sun are observed. The observation point is marked in Fig.1. The amount of solar radiation is also simultaneously measured on the surface of the ground near the fish-eye camera.

Fig. 2 shows time-series variation of (1) amount of solar radiation, (2) saturation of the image, (3) hue of the image and (4) brightness of the image at the same time.
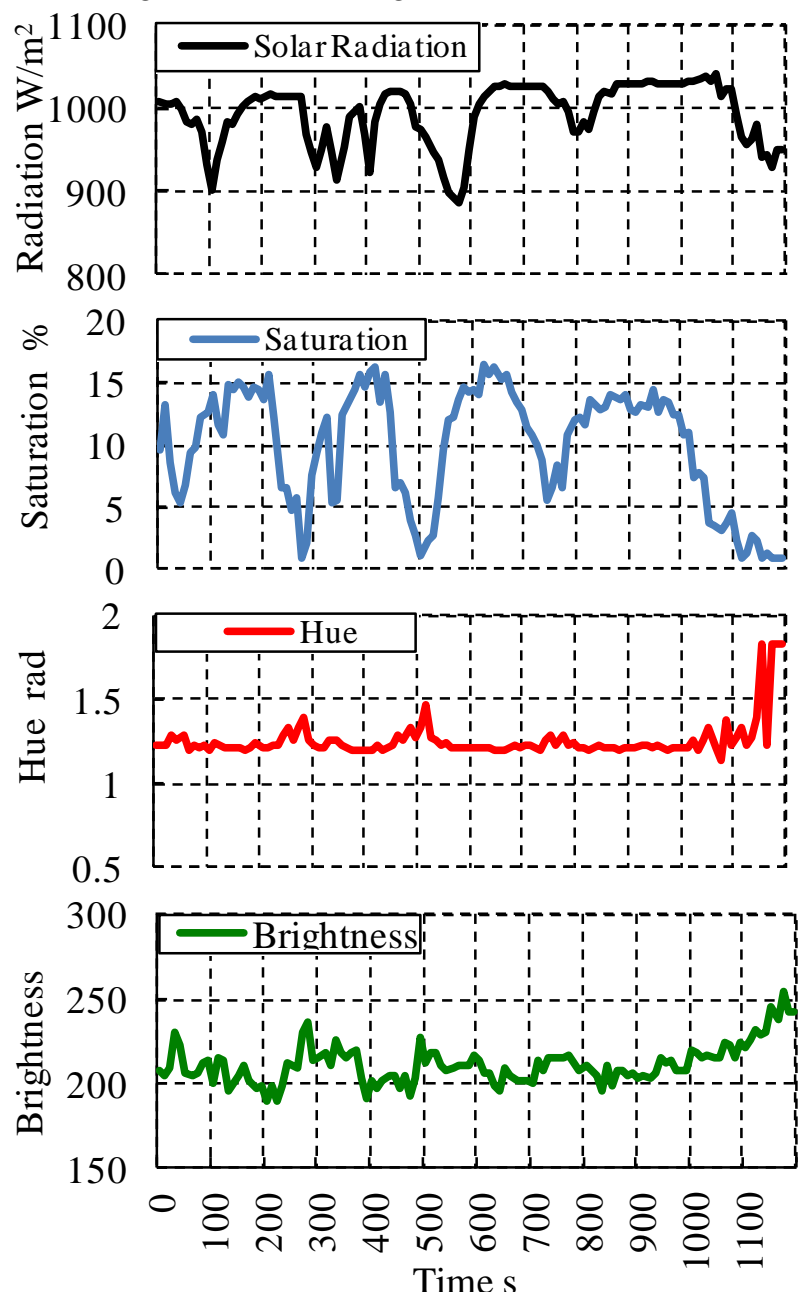

Fig.2 Time series of solar radiation, saturation, hue and brightness
The data is measured in a campus of Waseda University on May 13, 2012. The wave form of saturation of a point of the photo shows similarity with solar radiation measured on the ground. The event did not simultanously. The behavior of solar radiation is 2 minutes behind(lag) the behavior of saturation of image. This fact means that we can predict PV output by observing a processed fisheye photo image.

The observation point on the photo image is selected as an example in this analysis. If we want to forecast the PV output, more points around the sun should be observed. If the clouds goes to the lower part from the upper part of the photograph, useful observation points are located on the upper side of the sun.

\section{B. Correlation functions for forecasting}

In this section, more detailed approach to forecast the PV output is discussed. Fig. 3 shows the image of the photo around the sun. The image near to the sun is fogged by halation. Although the data near the sun seems to be useful, those are meaningless because of halation. If clouds move along straight line, the image information of point $\mathrm{A}, \mathrm{B}$ and $\mathrm{C}$ is useful. Cloud2 will cover the sun after $\mathrm{t}=2 \Delta \mathrm{t}$, if the saturation of image at point $\mathrm{B}$ is low. The image information of point $\mathrm{D}$ is useless, unless the movement of clouds becomes dotted line.

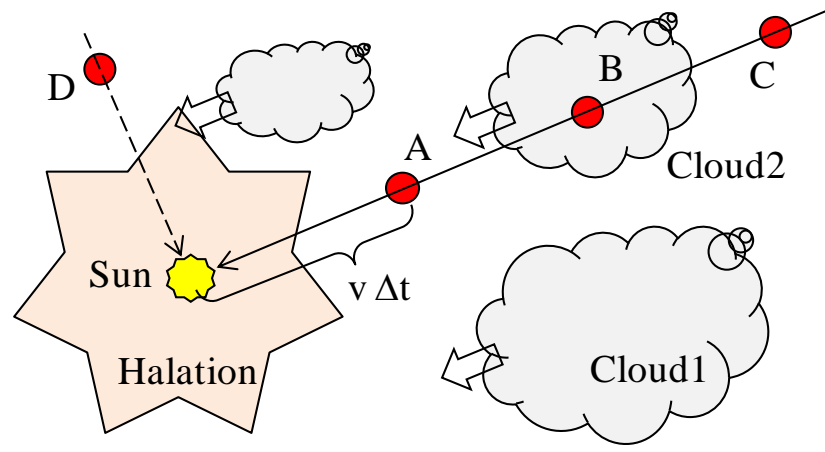

Fig3. Photo image and movement of cloud

Although we have discussed the movement of clouds, we do not identify clouds directly, since their shape and size may change. Instead, the correlation functions are calculated at all image points as in Eq. 1. If $\mathrm{R}(\tau)$ is large at certain points of photo, the saturation of images at those points can be used to predict the PV output of $\tau$ seconds ahead.

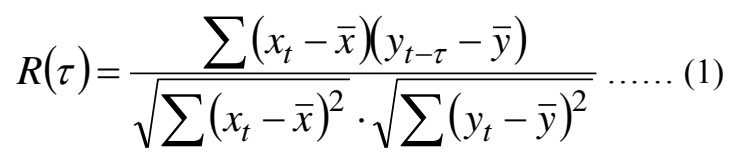

$R(\tau)$ :Correlation function

$$
\begin{aligned}
& \tau: \text { Time lag } \\
& x_{i}: \text { Amount of solar radiation (at time-i) } \\
& \left.y_{i}: \text { Saturation of image (at time }-\mathrm{i}\right)
\end{aligned}
$$

The time lag $\tau$ has important information. Fig.4 illustrates the image of correlation $\mathrm{R}(\tau)$ and $\tau$. If an observation point is close to the sun (e.g., point-A in Fig.3), the value of $\mathrm{R}$ will be large against smaller $\tau$. On the contrary, if an observation point is away from the sun 
(e.g., point-C in Fig. 3), the value of $\mathrm{R}$ will be large against larger $\tau$.

The correlation function $\mathrm{R}(\tau)$ is calculated using the measured data. Fig.5 shows the position of the sun and target area that is surrounded by a red rectangular. Grids are added to indicate the accurate position in latter process. Essentially, the target area should cover the sun, if we want to forecast the PV output in any cases. In this stage, however, the limited area is selected to avoid the treatment of halation.

\section{Point close to the sun ( Point-A)}
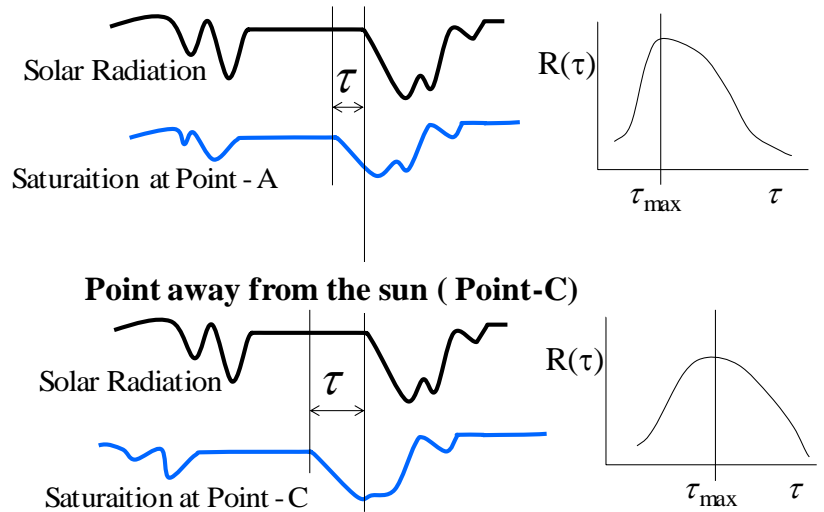

Fig4. Image of correlation between the amount of solar radiation and saturation with time lag $\tau$

\section{The correlation of the waveform of the amount of solar radiation and saturation}

Fig.6 shows the graph of the result analyzed correlation of the amount of solar radiation and saturation at each point. In this case, the sun is located in the south-east direction. The peak point is moving south-east if time lag $\tau$ becomes smaller. It is also easy to image the movement of cloud by tracing the hill of the Fig.6.

Fig.7 shows the graph of the result analyzed correlation of the amount of solar radiation and saturation. The time lag $\tau$ is variable and $\tau \max$ is used there.

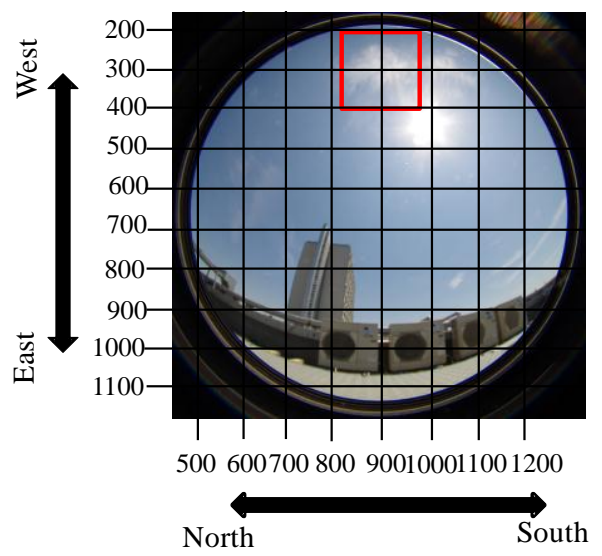

Fig.5 Position of the sun and the target area in whole sky image

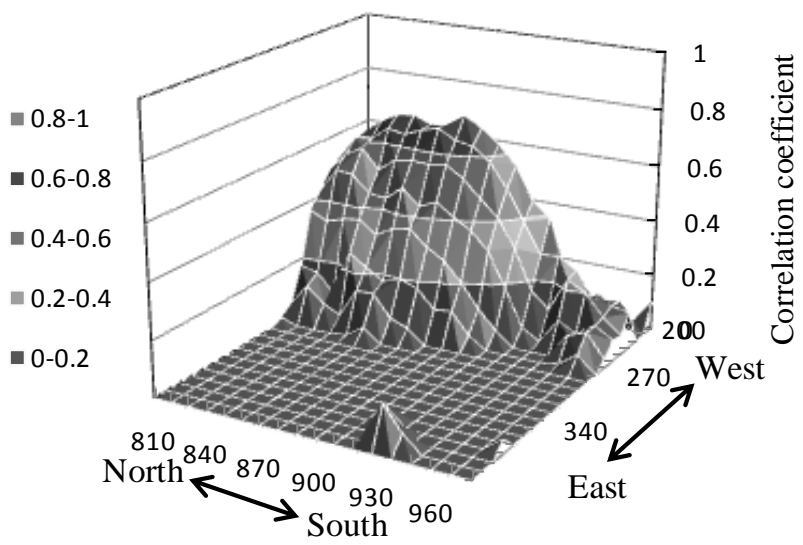

(a) Time lag $\tau=3$ minutes

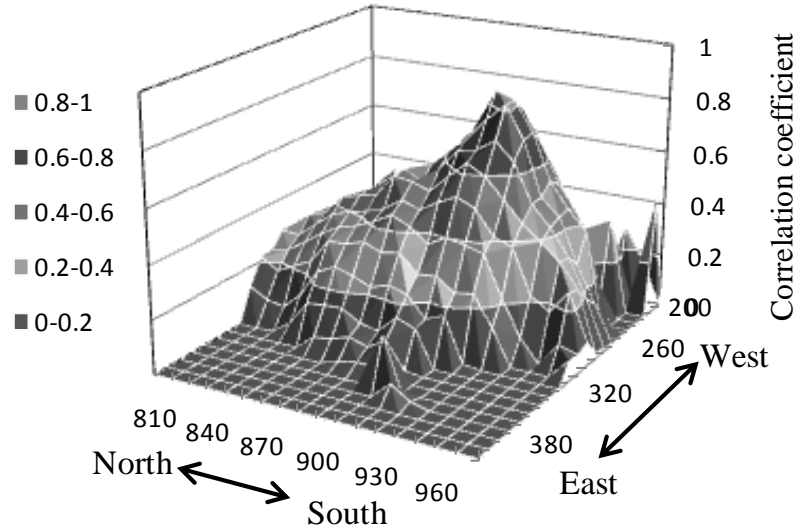

(b) Time lag $\tau=2$ minutes

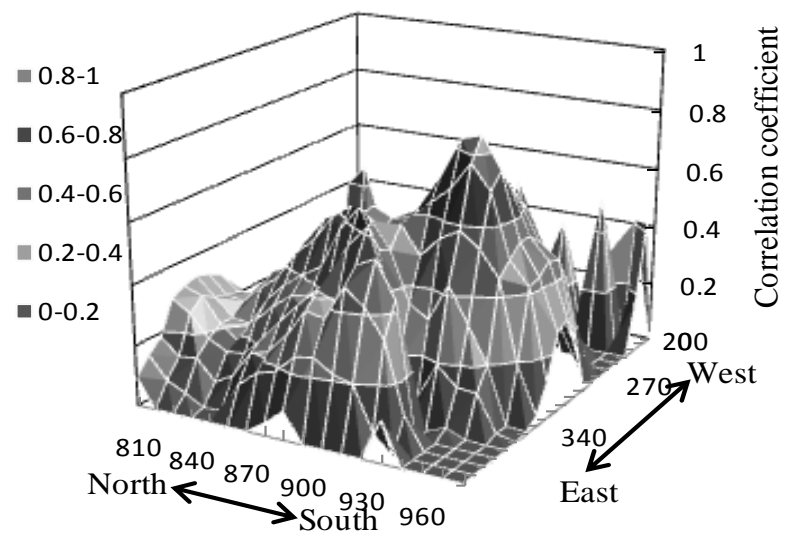

(c) Time lag $\tau=1$ minutes

Fig6. Calculated correlation $\mathrm{R}(\tau)$ with various time lag $\tau$

By varying the time lag $\tau$ from 10 seconds to 3 minutes, correation coefficient $\mathrm{R}(\tau)$ is scanned, and its maximum value is plotted in Fig. 7 .

Halation is difficult to treat. Halation is the phenomenon when a strong light is radiated, irradiation position is blurred and it is understood as white on the image. Therefore we should eliminate the area of halation from the evaluation of correations.

Fig. 8 shows the result of $\tau$-max that gives maximum $\mathrm{R}(\tau)$. This fugure shows the tendency of $\tau$-max well. 


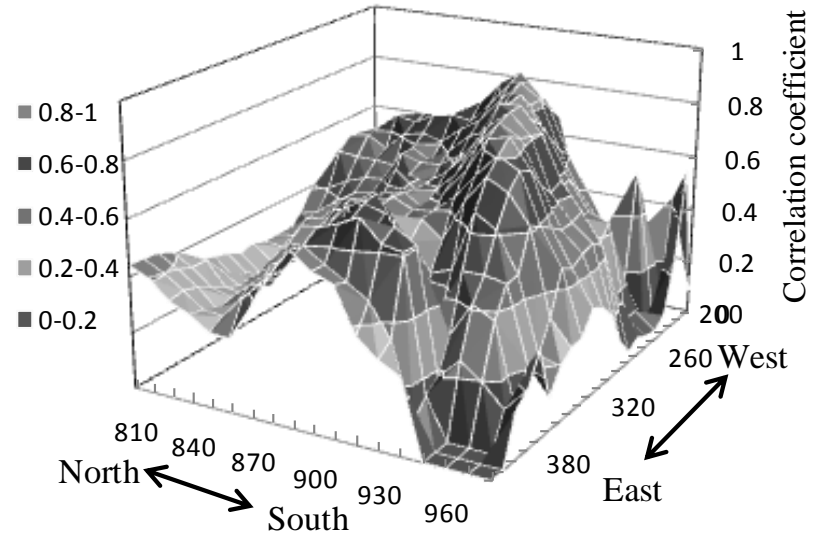

Fig.7 The correlation of the amount of solar radiation and saturation (time lag is variable. $\tau$-max is used.)

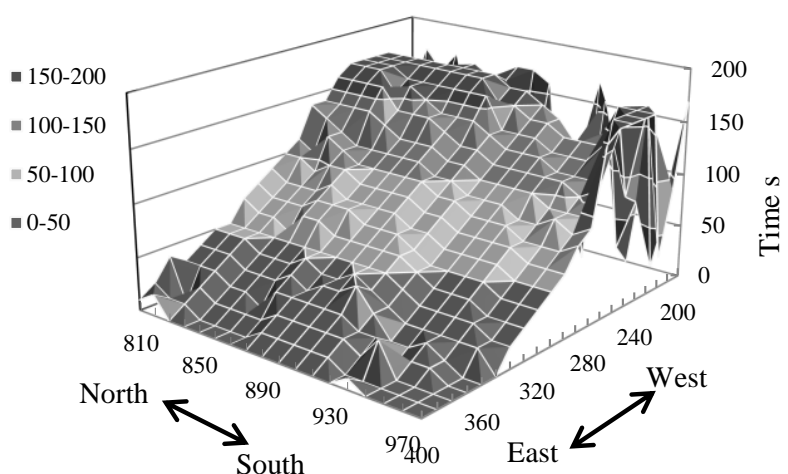

Fig.8 $\tau$-max that gives maximum $\mathrm{R}(\tau)$

\section{The correlation between solar radiation and saturation in cloudy weather}

Fig.9 shows the position of the sun and target area which is surrounded by a rectangular shape. In this case, the sky is overcast with dark clouds. The sun is located in the center of this image, and the clouds are moving from northwest to sotheast. Fig.10 shows the correlation $\mathrm{R}(\tau)$ by changing time lag $\tau$ on a cloudy day.

Fig.11 and Fig.12 show the maximum value of correlation by varying the time lag $\tau$ from 10 seconds to 3 minutes and the time lag gives maximum value in each point.The correlation on the cloudy day is lower than the sunny day, because clouds are heavy and widespread. Even if the time lag becomes small, the strong correlations are not shown. Hence, it is difficult to comprehend the movement of clouds on an overcast day from correlation between solar radiation and saturation in a sky image. Other elements or approaches are neccesary to forecast solar radiation on a cloudy day. However, the network/grid has small negative impact, since the fluctuation of solar radiation is small on an overcast day. Therefore, it is important to prepare different approaches for forecasting in various weather conditions.

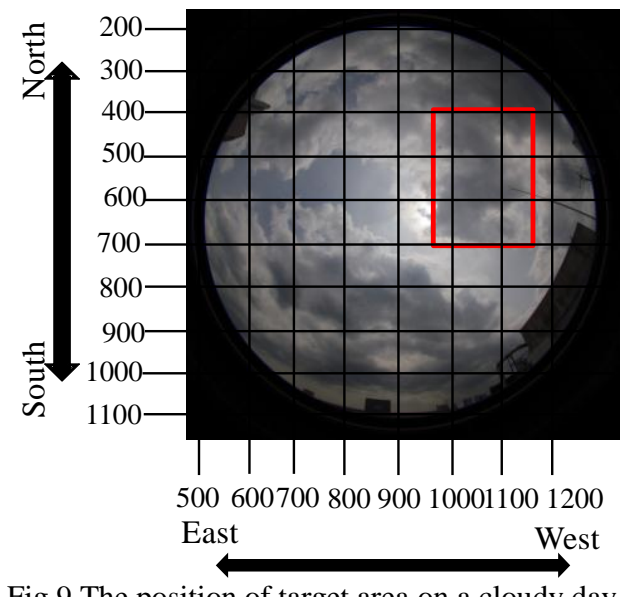

Fig.9 The position of target area on a cloudy day

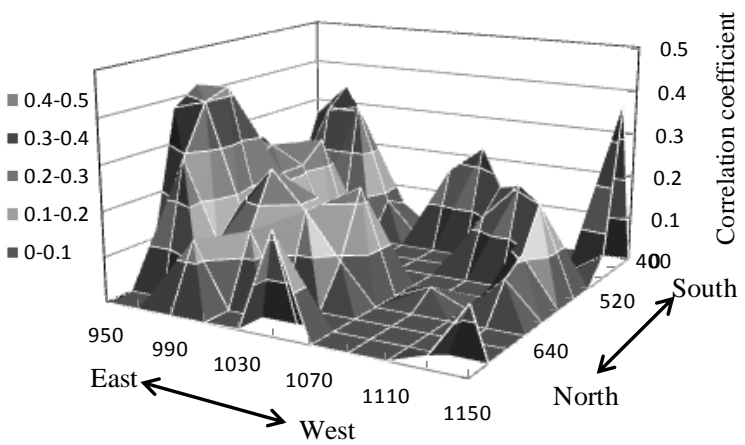

(a) Time lag $\tau=3$ minutes

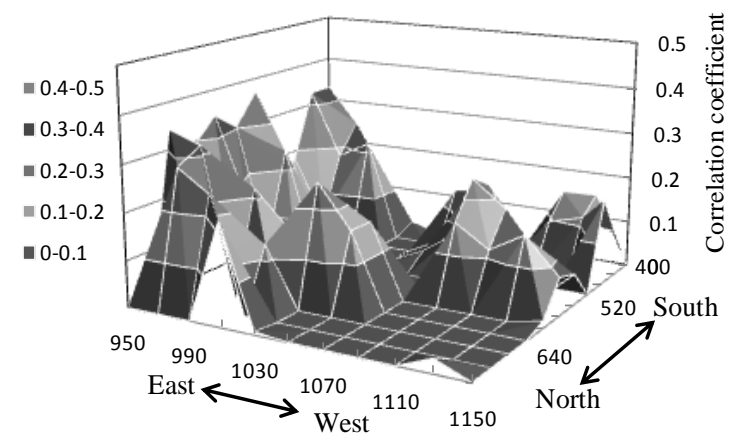

(b) Time lag $\tau=2$ minutes

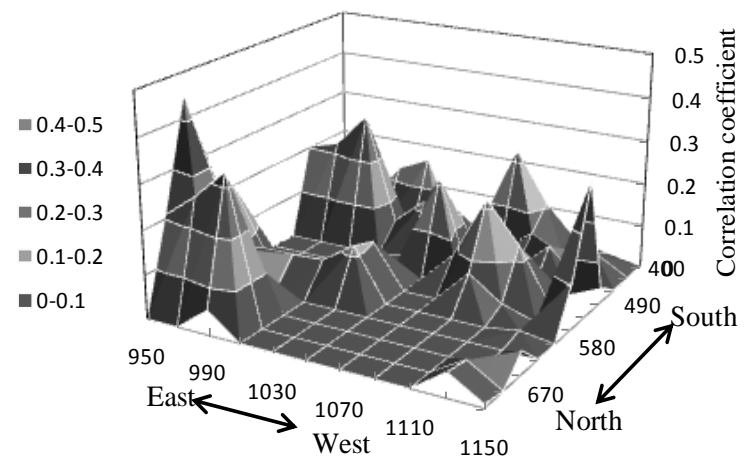

(c) Time lag $\tau=1$ minutes

Fig. 10 Correlation $\mathrm{R}(\tau)$ with various time lag $\tau$ on a cloudy day 


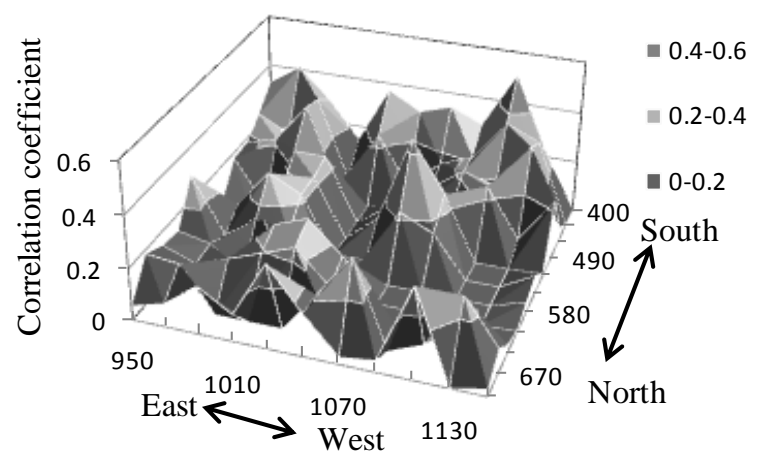

Fig.11 The correlation of the amount of solar radiation and saturation on an overcast day

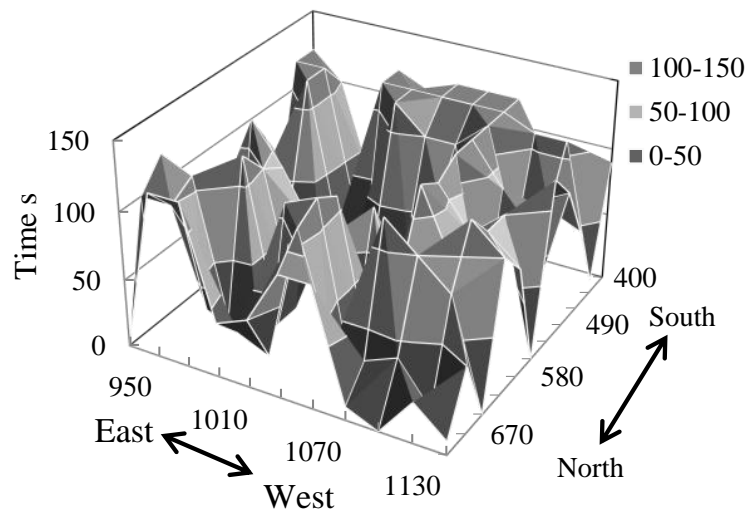

Fig.12 $\tau$-max that gives maximum $\mathrm{R}(\tau)$ on an overcast day

\section{Conclusion}

In this paper, we compared the amount of solar radiation and each element when a cloud passed through one point on a sunny day. Moreover we extracted several points around the sun and analyzed the correlation coefficient of the amount of solar radiation and the saturation. There are similarities between the waveform of the amount of solar radiation and the saturation. To analyze the correlation coefficient of both, the correlation coefficient of a sunny day is higher than one of cloudy days and can analyze well. On the other hand, the correlation coefficient on a cloudy day was low and cloud was not analyzed because prediction time was inconsistent. We are planning to measure the amount of global solar radiation and take a hemispherical photograph. Then we pay attention to plot the sun on the central position of the photograph and consider analyzing photograph of a cloudy day.

\section{Reference}

[1] K. Otani, T. Saitoh, I. Tsuda, K. Kurokawa, "SOLAR ENERGY MAPPING BY USING CLOUD IMAGES", IEEE First World Conference on Photovoltaic Energy Conversion 1994, Vol. 1, 805-808

[2] T. Katagi, S. Yamamoto, S. Hirayama, Y. Yamanouchi, JaeShik Park, T. Hashimoto, et al, "Basic research on the estimation of instantaneous power of photovoltaic system by using camera images", 2nd World Conference and Exhibition on Photovoltaic Solar Energy Conversion, Vol.2, pp.2092-2095 (1998-7)

[3] H. Taniguchi, K. Otani, K. Kurokawa, "THE MOTIONAL ANALYSIS OF CLOUD ALBEDO PATTERNS BY USING GMS IMAGES", The 28th IEEE Photovoltaic Specialists Conference

[4] T. Hashimoto, Y. Nagakura, "Prediction of Output Power
Variation of Solar Power Plant by Image Measurement of Cloud Movement", Journal of Advanced Research in Physics, Vol.2, No.2, 2011

[5] Yazmin Najera, Doug R. Reed, and W. Mack Grady, "IMAGE PROCESSING METHODS FOR PREDICING THE TIME OF CLOUD SHADOW ARRIVALS TO PHOTOVOLTAIC SYSTEMS",2011.

[6] LONG Zhi-yong, YI Xin, "Study on inversing cloud motion wind from visible and infraredsatellite image",Second IITA International Conferrence on Geoscience and Remote Sensing, 2010.

[7] Purdom J F W, "Detailed cloud motions from satellite imagery taken at thirty second, one and three minute intervals", Proc. to the 3rd International Wind Workshop. Ascona, Switzerland, 10-12 June, 1996. EUM P-1:137-145.

[8] Wang Z H, Browning K A and Kelly G A. Verification of the Tracking Technique Used in anExperimental Cloud Motion Wind Inferring System. JCMM Report. University of Reading, 1997.

\section{Biographies}

Yusuke Imanishi was born in Kochi, Japan, on May 16, 1989. He received B.S. degree in electrical engineering from Waseda University, Tokyo, Japan, in 2012. He is now a graduate student at the Graduate School of Environment and Energy Engineering in Waseda University in Japan. His research interest is the short period forecasting PV output using Hemispherical Photograph.

Ryo Watanabe was born in Kanagawa, Japan, on December 7, 1988. He received B.S degree in electrical engineering from Waseda University, Tokyo, Japan, in 2011. He is now a graduate student at the Graduate School of Environment and Energy Engineering in Waseda University in Japan. His research interest is impact assessment of PV generation on power systems.

Yusuke Hida was born in Kagoshima, Japan, on February 9, 1986. He graduated from department of electrical engineering in Meisei University, Tokyo Japan, in 2008. He received the degree of M.S in environment and energy engineering from Waseda University in 2010. He is now Ph.D student at the graduate school of environment and energy engineering in Waseda University in Japan. His research interests are environmental issues of power systems.

Ryuichi Yokoyama (M'73, F'08) received the degrees of B.S., M.S. and Ph.D. in electrical engineering from Waseda University, Tokyo Japan, in 1968, 1970 and 1973, respectively. After being engaged in Mitsubishi Research Institute, from 1978 through 2006, he worked in the Faculty of Technology of Tokyo Metropolitan University, and since 2007, he has been a professor at the Graduate School of Environment and Energy Engineering in Waseda University, Japan. Prof. Yokoyama is a fellow of IEEE of USA and the IEE of Japan and members of SICE of Japan, CIGRE, etc.

Kenji Iba (M'78, F'03) received the B.S and the M.S and $\mathrm{Ph} . \mathrm{D}$. degrees in electrical engineering from Waseda University in 1978, in 1980 and in 1990, respectively. He joined Mitsubishi Electric Corp. in 1980. He has been a professor of Department of Electrical Engineering in Meisei University from 2004. Professor Iba is a fellow of IEEE and a senior member of IEEJ. 